\title{
Macrophage Scavenger Receptor (SR-A I/II) Deficiency Reduced Diet- induced Atherosclerosis in C57BL/6J Mice
}

\author{
Nobuo Kamada1, Tatsuhiko Kodama², and Hiroshi Suzuki ${ }^{1,3}$ \\ 'Pharmaceutical Technology Laboratory, Chugai Pharmaceutical Co., Ltd., Shizuoka, Japan. \\ ${ }^{2}$ Research Center for Advanced Science and Technology, University of Tokyo, Tokyo, Japan. \\ ${ }^{3}$ National Research Center for Profozoan Diseases, Obihiro University of Agriculture and Veterinary Medicine, obihiro, \\ Japan.
}

\begin{abstract}
The effects of SR-A I/II deficiency and a synthetic anti-oxidant BO-653 on a diet-induced atherosclerosis in $\mathrm{C} 57 \mathrm{BL} / 6 \mathrm{~J}$, an inbred strain known to be susceptible to diet-induced atherosclerotic lesion formation, were examined. Quantitative analysis of the extent of atherosclerotic lesions in the mice fed the high-fat diet revealed that the atherosclerotic lesion area in SR-A I/II mutants was significantly reduced by $70 \%$ compared to wild type mice. A similar level of lesion reduction (75\%) was found in wild type mice fed the high-fat diet supplemented with $0.6 \%$ BO-653 compared to those without BO-653. Thus, for $\mathrm{C57BL} / 6 \mathrm{~J}$ in the setting of prolonged exposure to a high-fat diet, defect of SR-A I/II expression is significantly protective against the development of atherosclerosis, as is the synthetic anti-oxidant BO-653. These results indicate that SR-A I/II has a crucial role in atherosclerotic lesion formation with uptake of oxidized-LDL in this mouse model.

Atheroscler Thromb, $2001 ; 8$ : 1-6.
\end{abstract}

Key words : Gene targeting, Genetic background, BO-653, Oxidized-LDL

\section{Introduction}

Scavenger receptor type A (SR-A I/II) is a trimeric integral membrane glycoprotein (1-3) and has multiple functions including the receptor-mediated uptake of modified lipoproteins (4), cell adhesion (5), phagocytosis (6) and host defense (7). Since SR-A I/II is widely expressed in macrophages and foam cells of atherosclerotic lesions $(8,9)$, the uptake of modified LDL by SR-A I/II has been proposed to play a key role in atherogenesis. The establishment of mice with targeted disruption of the SR-A I/II has provided an important insight into the role of the SR-A I/II in atherogenesis in vivo (7). Both SR-A I/II and apolipoprotein E double-

Address for correspondence: Hiroshi Suzuki, National Research Center for Protozoan Diseases, Obihiro University of Agriculture and Veterinary Medicine, Obihiro, Hokkaido 080-8555, Japan.

E-mail : hisuzuki@obihiro.ac.jp

Received November 10, 2000

Accepted for publication January 11, 2001. knockout mice demonstrate significantly reduced ( 60\%) lesion size compared to apo E knockout (7). Similarly, under a high fat and high cholesterol diet, mice deficient in both SR-A I/II and LDL receptors showed a significant reduction $(\sim 20 \%)$ in aortic lesion area compared to LDL receptor deficient mice (10). Although the reason for the more prominent effect of SR-A I/II disruption on atherosclerosis in the apo $\mathrm{E}$ knockout mice than in the LDL receptor knockout mice is still unknown, both these studies indicate a proatherogenic role for SR-A I/II. However, apo E3 Leiden transgenic mice with SR-A I/II deficiency showed a tendency of increase in lesion area with the development of more complex lesions (11). A possible interpretation of these conflicting results might be that apo $\mathrm{E}$ modulates the effect of SR-A I/II deficiency on atherosclerosis. However, more importantly, other factors such as the genetic backgrounds of mice should be examined in order to understand these results. All of the genetically manipulated mice used in the above studies were from mixed genetic background. Thus, we examined effects of SR-A I/II deficiency on the diet- 
induced atherogenesis in C57BL/6J mice, an inbred strain known to be susceptible to atherogenesis (12). Furthermore, the effects of SR-A I/II deficiency in combination with synthetic anti-oxidant treatment were also investigated with regard to the reduction of atherosclerotic lesions in C57BL/6J mice.

\section{Materials and Methods}

The genetic background of the SR-A I/II knockout mice (9) was replaced by backcrossing into C57BL/6J (CLEA Japan, Tokyo, Japan) for at least eight generations. In general, a congenic strain of mice is established by backcrossing for eight or twelve generations (13). Established homozygous mutant females of C57BL/6J genetic background (B6-SR-A I/II KO) and wild type C57BL/6J females, at 3 months of age, were fed with a high-fat diet or a standard laboratory chow containing $4.4 \%$ fat and $0.1 \%$ cholesterol (CE-2, CLEA Japan, Tokyo, Japan) ad lib. for 7 months. Thirteen to 15 females were supplied for each experimental group. The high-fat diet was a synthetic diet containing $1.25 \%$ cholesterol, $50 \%$ sucrose, $20 \%$ casein, $15 \%$ cocoa butter, $5 \%$ AIN mineral mixture, $4.95 \%$ cellulose, $1 \%$ AIN vitamin mixture, $1 \%$ cholin chloride, $1 \%$ corn oil, $0.5 \%$ cholic acid and $0.3 \% \mathrm{D}, \mathrm{L}$-methionine (CLEA Japan, Tokyo, Japan). When a synthetic anti-oxidant, 0.6\% BO-653 (14), was added to the high-fat diet, the concentration of cellulose was reduced to $4.35 \%$. The plasma concentrations of lipids and lipoproteins and body weights of the mice were monitored at four week intervals. Blood was sampled by retro-orbital puncture, and the plasma lipids and lipoproteins were measured using a Roche COBAS FARA II clinical chemistry analyzer (Roche, Basel, Switzerland) and high-performance liquid chromatography (HPLC; TSK-GEL, Tosoh, Tokyo, Japan). Mice were sacrificed under ether anesthesia and their hearts with aorta were removed. Atherosclerotic lesions were evaluated quantitatively according to the method described previously $(7,15)$. Briefly, the heart and vascular tree were fixed with $4 \%$ paraformaldehyde at $4^{\circ} \mathrm{C}$ for 4 hours. After washing with phosphate-buffered saline, they were embedded in OCT compound (TissueTek, 4583, Miles Laboratories, Elkhart, Indiana,USA) and frozen in liquid nitrogen. Frozen sections (6 $\mu \mathrm{m}$ thin) through the aortic valve were cut serially by cryostat. Each section was stained with Sudan IV (O 0100, TCI, Tokyo,Japan), counterstained with hematoxylin, and mounted with Aquatex (Merck, Darmstadt, Germany). Four sections were selected, and atherosclerotic lesions were measured using the Sigma Scan Pro software (Jandel Scientific Software, USA). The average of the four sections represents the aortic lesion size for each mouse.

All mice were housed in polycarbonate cages (TP-106; $175 \times 245 \times 125 \mathrm{~mm}$, TP-102; $215 \times 320 \times 140 \mathrm{~mm}$, Toyoriko, Japan), and maintained as specific pathogen-free in a light-controlled (lights-on from 05:00 to 19:00) and air- conditioned room set at $24 \pm 2^{\circ} \mathrm{C}$ and $55 \pm 10 \%$ humidity. Mice used in this study were cared for and used under the Guiding Principles for the Care and Use of Research Animals promulgated by Chugai Pharmaceutical, Shizuoka, Japan.

Statistical significance of the data was evaluated by use of the Student's $t$-test and Steel-Dwass test (Tukey type non-parametric comparison). A $p$ value of $<0.05$ was regarded as significant.
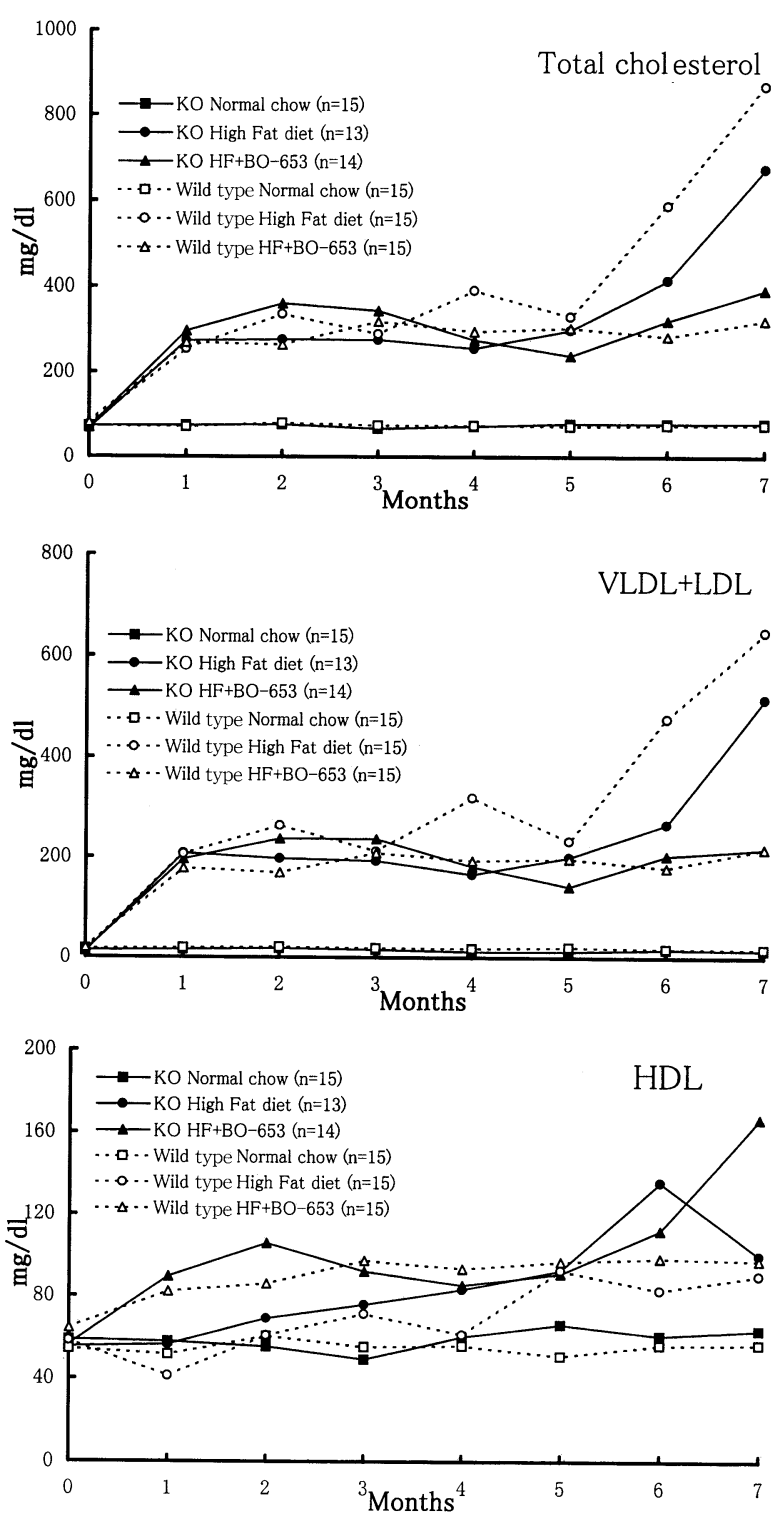

Fig. 1. Plasma cholesterol, VLDL $+\mathrm{LDL}$ and HDL levels in SR-A I/II deficient C57BL6/J female mice fed with an atherogenic diet. SR-A I/II mutant and wild type mice were maintained on a high-fat diet with or without $0.6 \%$ BO-653 for 7 months. 


\section{Results}

After one month of the atherogenic diet with or without BO-653 supplementation, both wild type and SR-A I/II mutant mice were hypercholesterolemic (Fig. 1). The total plasma cholesterol and VLDL+LDL cholesterol levels of these mice increased dramatically after six months of the atherogenic diet. These significant increases of cholesterol levels were inhibited by BO-653 supplementation in each genotype. HDL cholesterol measurements of the mice fed the atherogenic diet did not show significant differences between the genotypes. BO-653 significantly increased HDL cholesterol levels from one to three months after the diet supplementation in each genotype ( $p<0.01$ wild type ; $p<0.03$ SR-A I/II KO ; Fig. 1). As shown in Figs. 2 and 3, quantitative analysis of the extent of atherosclerotic lesions in the mice fed the atherosclerotic diet revealed that the mean atherosclerotic lesion area $\left(\mu \mathrm{m}^{2} \pm \mathrm{SD}\right)$ in SR-A I/II mutants was reduced by $70 \%$ compared to wild type mice $(32,553 \pm$ 2,484 vs $107,777 \pm 3,513, p=0.000004$; Fig. 2). A similar level of lesion reduction (75\%) was found in wild type mice fed the atherogenic diet supplemented with 0.6\% BO-653 compared to those without BO-653 $(26,574 \pm 1,896$ vs $107,777 \pm 3,513, \quad p=0.0000005$; Fig. 2). Thus, for $\mathrm{C} 57 \mathrm{BL} / 6 \mathrm{~J}$ in the setting of prolonged exposure to an atherogenic diet, the defect of SR-A I/II expression is significantly protective against the development of atherosclerosis, as is the synthetic anti-oxidant BO-653. Furthermore, SR-A I/II deficiency in combination with BO653 treatment had a more prominent effect than the SRA I/II deficiency alone in the mice fed the atherogenic diet
( $p=0.04$, Fig. 2). There was no statistical correlation between the plasma VLDL+LDL cholesterol level and atherosclerotic lesion size in each experimental group $\left(r^{2}=0.0495\right.$ B6-SR-A I/II KO; $r^{2}=0.2946$ B6-SR-A I/II $\mathrm{KO}$ with $\mathrm{BO}-653 ; r^{2}=0.0001$ wild type $; r^{2}=0.002$ wild type with BO-653; Fig. 4). Also, no significant differences in plasma triglyceride levels and body weights were found between the genotypes of mice fed with the atherogenic diet throughout the experiment (data not shown). Neither wild type nor SR-A I/II mutant mice fed with a normal chow exhibited atherosclerotic lesions (Figs. 2, 3).

\section{Discussion}

The results of this study clearly demonstrated that SRA I/II expression promotes a diet-induced atherosclerosis in vivo. We have previously reported that SR-A I/II deficiency reduced the extent of atherosclerotic lesions in apo $E$ (7) and LDL receptor knockout mice (10). In contrast, apo E3 Leiden transgenic mice with SR-A I/II disruption showed an increase of $80 \%$ in lesion size with more complex lesions (11). These results led us to the interpretation that apo $E$ might modulate the effect of SRA I/II deficiency on atherogenesis (11). However, these conflicting results may be explained by the mixed genetic background of the mice, since all the genetically manipulated mice used the previous studies were hybrid strains mixed with ICR, 129/SvJ and C57BL/6. As expected, SR-A I/II disrupted mice of the C57BL/6J background were resistant to diet-induced atherosclerosis, with a reduction of $70 \%$ in lesion size compared to wild type animals (Fig. 2). Our finding indicates that the

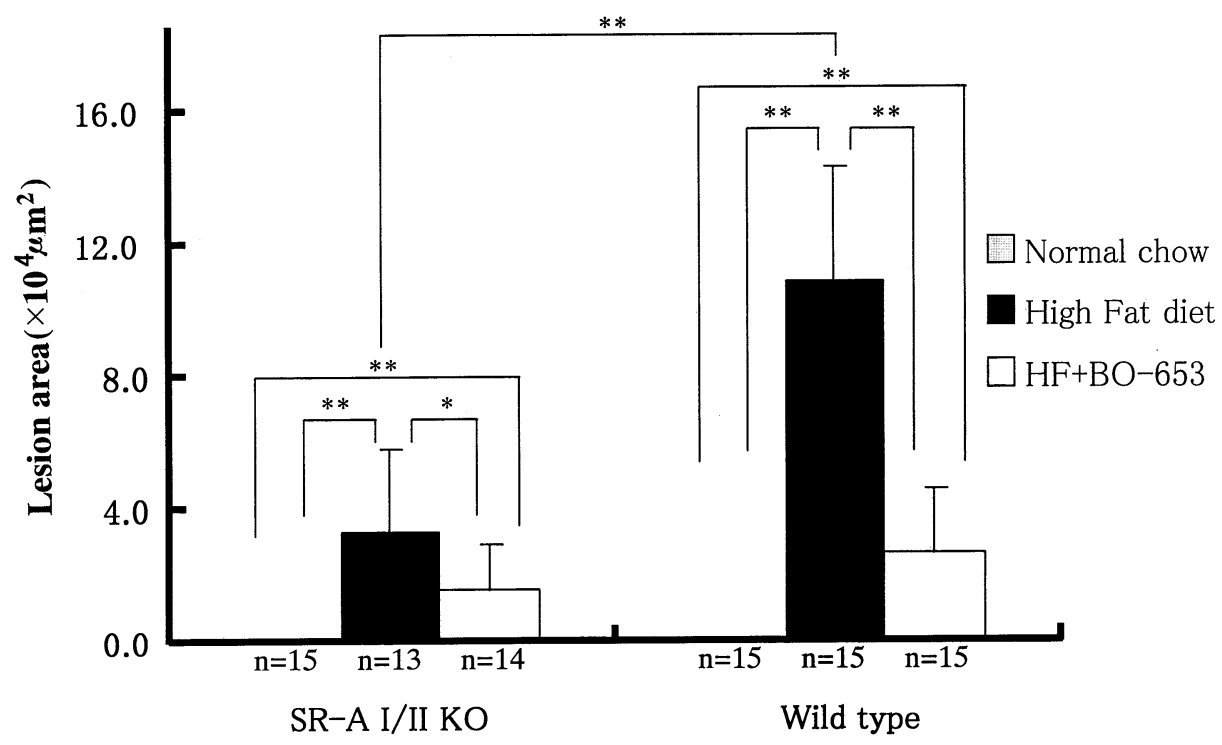

Fig. 2. Effect of SR-A I/II deficiency and a synthetic anti-oxidant BO-653 on diet-induced atherosclerosis in C57BL/6J mice. SR-A I/II mutant and wild type mice were maintained on a high-fat diet with or without $0.6 \% \mathrm{BO}-653$ for 7 months. All results are shown as mean $\pm \mathrm{SD}$. ${ }^{*}: p<0.05,{ }^{* *}$ : $p<0.01$. 


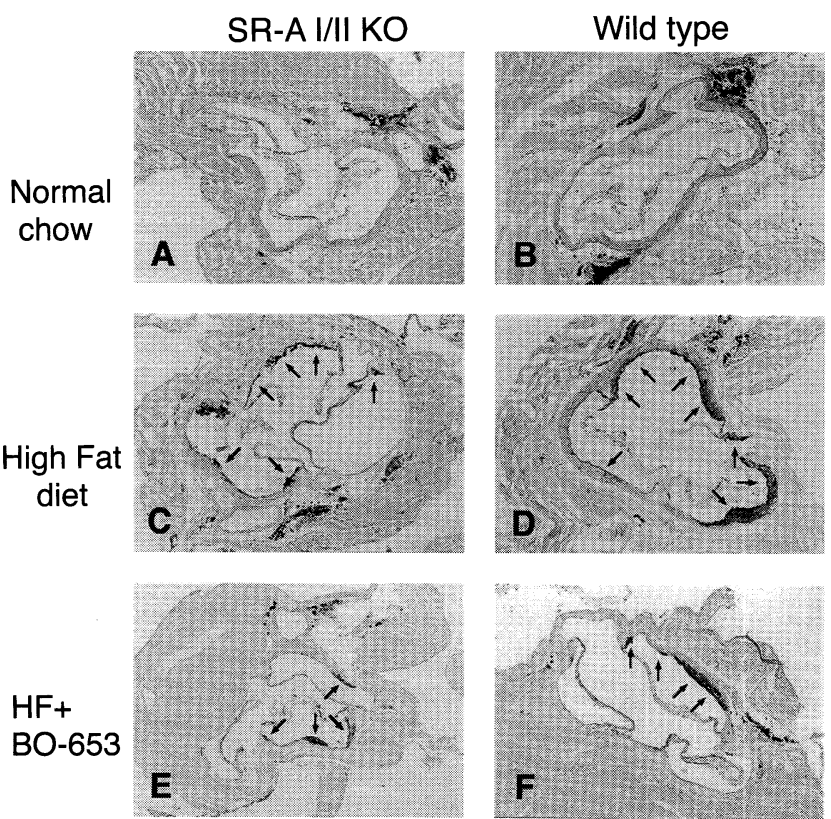

Fig. 3. Representative cross sections of aortic valves in hearts of mice maintained on a high-fat diet with or without $0.6 \%$ BO-653 for 7 months. Atherosclerotic lesions stained with Sudan IV are shown by arrows.

mixed genetic background may have caused a smaller reduction in lesion size in LDL receptor and SR-A I/II double knockout mice (10) and an enhanced effect of SRA I/II deficiency on atherogenesis in apo E3 Leiden transgenic mice (11). The presence or absence of apo $E$ does not seem to modulate the effect of SR-A I/II disrup- tion on atherogenesis, since the reductions in atherosclerotic lesions in SR-A I/II mutants with normal apo $E$ (Fig. 2) were similar to those in SR-A I/II with apo E disruption (7). Recently, it has been reported that CD36 disruption protects against atherogenesis in apo $\mathrm{E}$ knockout mice (16). Although CD36 is different from SR-A I/II in structure, CD36 also mediates binding and uptake of modified lipoproteins and has been implicated in foam cell formation, similar to SR-A I/II functions. Both CD36 and apo $E$ deficient macrophages showed a $50 \%$ reduction in uptake of acetyl-LDL and a $60 \%$ reduction in uptake of oxidized-LDL (16). We have previously reported that the uptake of acetyl-LDL in SR-A I/II deficient macrophages was reduced by $65-80 \%$, whereas the uptake of oxidizedLDL was reduced by $30-50 \%(7,17,18)$. Sakai et al. (19) reported that SR-A I/II disruption in macrophages showed a $70 \%$ reduction in the uptake of oxidized-LDL. The specificity for uptake of modified LDL and the extent of reduction in atherosclerosis in apo $E$ knockout mice with SR-A I/II or CD36 seem to be similar $(7,16)$. However, since the extent of oxidation may be quite different from preparation to preparation or from laboratory to laboratory, even if the procedure for oxidation is the same, careful interpretation of studies of the interaction of oxidized-LDL with receptors is clearly required.

The effect of the synthetic anti-oxidant, BO-653, was also remarkable for diet-induced atherosclerosis in C57BL/6J regardless of SR-A I/II expression, as previously reported (14). SR-A I/II mutant mice fed the atherogenic diet with $0.6 \%$ BO-653 showed a significant decrease in lesion size (by $54 \%$ ) compared with those without BO-653 supplementation $(p=0.04)$. These find-

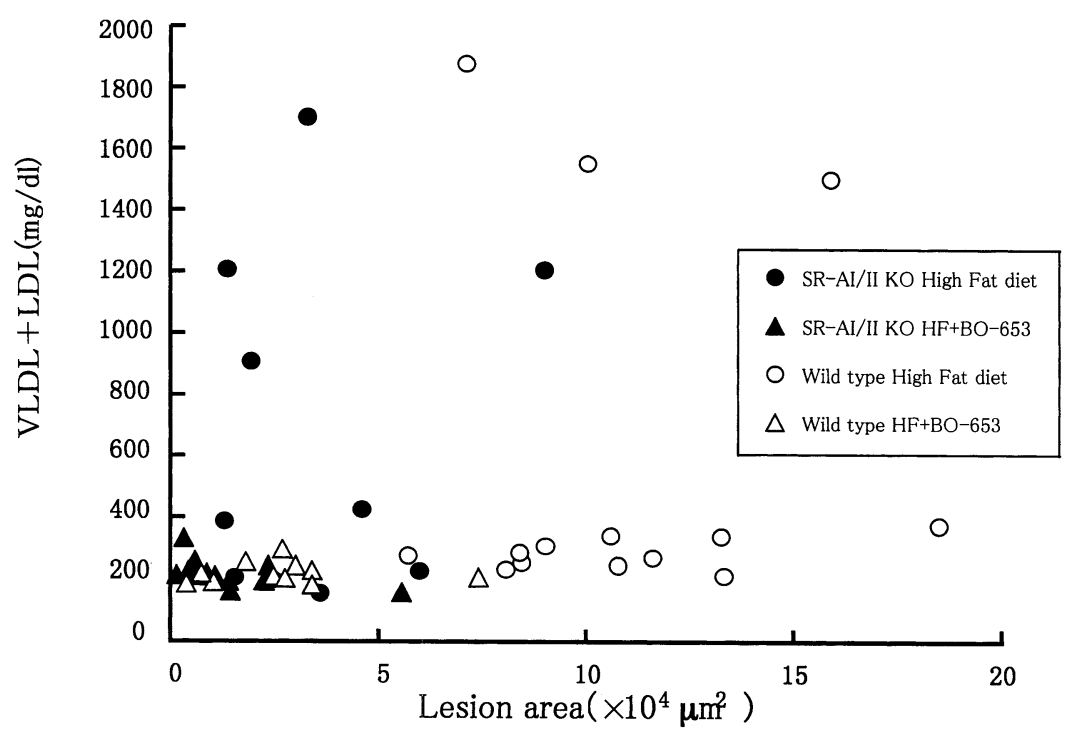

Fig. 4. Correlation between plasma VLDL + LDL cholesterol levels and atherosclerotic lesions in SRA I/II deficient C57BL6/J female mice maintained on a high-fat diet with or without $0.6 \%$ BO-653. Lesion sizes of aortic atherosclerosis are plotted against plasma VLDL+LDL cholesterol levels after 7 months of the atherogenic diet. 
ings indicate that the inhibition of lipoprotein oxidation is more effective for protection against atherosclerotic lesion formation than the elimination of SR-A I/II activity. The difference in lesion size between SR-A I/II mutants fed the atherogenic diet with or without BO-653 does not seem to reflect the inhibitory effect of BO-653 for significant increases in plasma VLDL + LDL cholesterol levels in mice fed with the atherogenic diet at and after 6 months (Figs. 1,2), since no statistical correlation was found between the plasma VLDL+LDL cholesterol level and atherosclerotic lesion size (Fig. 3). There are considerable strain differences in diet-induced atherosclerosis susceptibility in mice $(20,21)$. DBA $/ 2, C 57 B L / 6 J$ and $\mathrm{C} 57 \mathrm{BL} / 10$ are known to be susceptible for diet-induced atherosclerosis. The genetic locations of atherosclerosis susceptibility genes have been mapped such as Ath-1 (22), Ath-2 (23) and Ath-3 (24). Although the best characterized of these genetic loci was Ath-1, for the presumptive gene at that genetic position that controls atherosclerosis susceptibility, recent quantitative trait locus mapping (QTL) has failed to detect the Ath-1 locus $(25,26)$. The high susceptibility of C57BL/6J to dietinduced atherosclerosis might be explained by the higher activity of SR-A I/II or CD36, however, variation of SR-A I/II or CD36 activity among inbred mouse strains has not been examined yet.

In conclusion, these results indicate that SR-A I/II has a crucial role in atherosclerotic lesion formation with uptake of oxidized-LDL in this mouse model.

Acknowledgements : We wish to thank H. Kaise, T. Iwata and S. Uchida for technical assistance.

\section{References}

(1) Kodama T, Reddy P, Kishimoto $C$, and Kriger M: Purification and characterization of a bovine acetyl low density lipoprotein receptor. Proc Natl Acad Sci USA, 85 : 9238-9242, 1988

(2) Kodama T, Freeman M, Rohrer L, Zabrecky J, Matsudaira $P$, and Krieger $M$ : Type I macrophage scavenger receptor contains alpha-helical and collagen-like coiled coils. Nature, $343: 531-535,1990$

(3) Rohrer L, Freeman M, Kodama T, Penman M, and Krieger $\mathrm{M}$ : Coiled-coil fibrous domains mediate ligand binding by macrophage scavenger receptor type II. Nature, 343: 570-572, 1990

(4) Brown MS and Goldstein JL: Lipoprotein metabolism in the macrophage : implications for cholesterol deposition in atherosclerosis. Annu Rev Biochem, 52: 223-261, 1983

(5) Fraser I, Hughes D, and Gordon S: Divalent cation-independent macrophage adhesion inhibited by monoclonal antibody to murine scavenger receptor. Nature, 364: 343-346, 1993

(6) Platt N, Suzuki H, Kurihara Y, Kodama T, and Gordon S: Role for the class $A$ macrophage scavenger receptor in the phagocytosis of apoptotic thymocytes in vitro. Proc
Natl Acad Sci USA, 93: 12456-12460, 1996

(7) Suzuki H, Kurihara Y, Takeya M, Kamada M, Kataoka M, Jishage K, Ueda O, Sakaguchi H, Higashi T, Suzuki T, Takashima Y, Kawabe Y, Cynshi O, Wada Y, Honda M, Kurihara $\mathrm{H}$, Aburatani $\mathrm{H}$, Doi T, Matsumoto A, Azuma S, Noda T, Toyoda Y, Itakura H, Yazaki Y, Horiuchi S, Takahashi K, Kruijt JK, van Berkel TJC, Steinbrecher UP, Ishibashi S, Maeda N, Gordon S, and Kodama T: A role for macrophage scavenger receptors in atherosclerosis and susceptibility to infection. Nature, 386: 292-296, 1997

(8) Matsumoto A, Naito M, Itakura $\mathrm{H}$, Ikemoto $\mathrm{S}$, Asaoka $\mathrm{H}$, Hayakawa I, Kanamori H, Aburatani H, Takaku F, Suzuki H, Kobari Y, Miyai T, Takahashi K, Choen EH, Wydro R, Housman DE, and Kodama $\mathrm{T}$ : Human macrophage scavenger receptors: Primary structure, expression, and localization in atherosclerotic lesions. Proc Natl Acad Sci USA, 87 : 9133-9137, 1990

(9) Naito M, Suzuki H, Mori T, Matsumoto A, Kodama T, and Takahashi K: Coexpression of type I and type II human macrophage scavenger receptors in macrophages of various organs and foam cells in atherosclerotic lesions. Am J Pathol, 141: 591-599, 1992

(10) Sakaguchi H, Takeya M, Suzuki H, Hakamata $H$, Kodama T, Horiuchi S, Gordon S, van der Laan, Luc JW, Kraal G, Ishibashi S, Kitamura $\mathrm{N}$, and Takahashi $\mathrm{K}$ : Role of macrophage scavenger receptors in diet-induced atherosclerosis in mice. Lab Invest, 78: 423-434, 1998

(11) de Winther MP, Gijbels MJ, van Dijk KW, van Gorp PJ, Suzuki H, Kodama T, Frants RR, Havekes LM, and Hofker $\mathrm{MH}$ : Scavenger receptor deficiency leads to more complex atherosclerotic lesions in APOE3 Leiden transgenic mice. Atherosclerosis, 144 : 315-321, 1999

(12) Ishida BY and Paigen B : Genetic Factors in Atherosclerosis: Approaches and Model Systems, vol 12, pp 189 222, 1989

(13) Yamada J : Genetic control of laboratory animals. In: Laboratory Animals Handbook. ed by Nagasawa $H$, Fujiwara K, Maejima K, Matsushita H, Yamada J, and Yokoyama A, pp 7-17, Yokendo, Tokyo, 1983 (in Japanese)

(14) Cynshi O, Kawabe $Y$, Suzuki T, Takashima $Y$, Kaise H, Nakamura M, Ohba Y, Kato, Y, Tamura K, Hayasaka A, Higashida A, Sakaguchi H, Takeya M, Takahashi K, Inoue K, Noguchi N, Niki E, and Kodama T: Antiatherogenic effects of the antioxidant BO-653 in three different animal models. Proc Natl Acad Sci USA, 95 : 1012310128, 1998

(15) Zhang SH, Reddick RL, Burley B, and Maeda N : Dietinduced atherosclerosis in mice heterozygous and homozygous for apolipoprotein $E$ gene disruption. J Clin Invest, 94 : 937-945, 1994

(16) Febbraio M, Podrez EA, Smith JD, Hajjar DP Hazen SL, Hoff HF, Sharma K, and Silverstein RL: Targeted disruption of the class $\mathrm{B}$ scavenger receptor CD36 protects against atherosclerotic lesion development in mice. J Clin Invest, 105: 1049-1056, 2000

(17) Lougheed M, Lum CM, Ling W, Suzuki H, Kodama T, and Steinbrecher $\mathrm{U}$ : High affinity saturable uptake of oxidized low density lipoprotein by macrophages from mice lacking the scavenger receptor class A type I/II. J Biol Chem, 272 : 12938-12944, 1997

(18) Terpstra V, Kondratenko N, and Steinberg D: Macro- 
phages lacking scavenger receptor A show a decrease in binding and uptake of acetylated low-density lipoprotein and of apoptotic thymocytes, but not of oxidatively damaged red blood cells. Proc Natl Acad Sci USA, 94 : 8127-8131, 1997

(19) Sakai M, Miyazaki A, Hakamata H, Kodama T, Suzuki H, Kobori S, Shichiri M, and Horiuchi S: The scavenger receptor serves as a route for internalization of lysophosphatidylcholine in oxidized low density lipoprotein-induced macrophage proliferation. J Biol Chem, 271: 27346-27352, 1996

(20) Paigen B, Morrow A, Brandon C, Mitchell D, and Holmes $P$ : Variation in susceptibility to atherosclerosis among inbred strains of mice. Atherosclerosis, 57:65-73, 1985

(21) Qiao JH, Xie PZ, Fishbein MC, Kreuzer J, Drake TA, Demer LL, and Lusis AJ: Pathology of atheromatous lesions in inbred and genetically engineered mice. Arterioscler Thromb, 14 : 1480-1497, 1994

(22) Paigen B, Mitchell D, Reue K, Morrow A, Lusis AJ, and
LeBoeuf RC: Ath-1, a gene determining atherosclerosis susceptibility and high density lipoprotein levels in mice. Proc Natl Acad Sci USA, 84 : 3763-3767, 1987

(23) Paigen B, Nesbitt MN, Mitchell D, Albee D, and LeBoeuf $\mathrm{RC}$ : Ath-2, a second gene determining atherosclerosis susceptibility and high density lipoprotein levels in mice. Genetics, 122 : 163-168, 1989

(24) Stewart-Phillips JL, Lough J, and Skamene E : ATH-3, a new gene for atherosclerosis in the mouse. Clin Invest Med, 12: 121-126, 1989

(25) Hyman RW, Frank S, Warden $\mathrm{CH}$, Daluiski A, Heller R, and Lusis AJ: Quantitative trait locus analysis of susceptibility to diet-induced atherosclerosis in recombinant inbred mice. Biochem Genet, 32 : 397-407, 1994

(26) Machleder D, Ivandic B, Welch C, Castellani L, Reue K, and Lusis AJ : Complex genetic control of HDL levels in mice response to an atherogenic diet. Coordinate regulation of HDL levels and bile acid metabolism. J Clin Invest, 99: 1406-1419, 1997 